\title{
Polyvalent Melanoma Vaccine
}

National Cancer Institute

\section{Source}

National Cancer Institute. Polyvalent Melanoma Vaccine. NCI Thesaurus. Code C1633.

A cancer vaccine consisting of whole irradiated heterologous melanoma cells which express multiple melanoma-related antigens. Polyvalent melanoma vaccine may stimulate an antitumoral cytotoxic T-cell immune response in the host, resulting in inhibition of tumor cell proliferation and tumor cell death. (NCI04) 